\title{
Numerical Algorithms for Two-Dimensional Dry Granular Flow with Deformable Elastic Grains
}

\author{
Henry A. Boateng \\ Valjean Elander \\ Chao Jin \\ Yan $\mathrm{Li}$ \\ Paula Vasquez \\ Petri Fast
}

Final Report

IMA Mathematics in Industry Workshop IX

U.S. Department of Energy

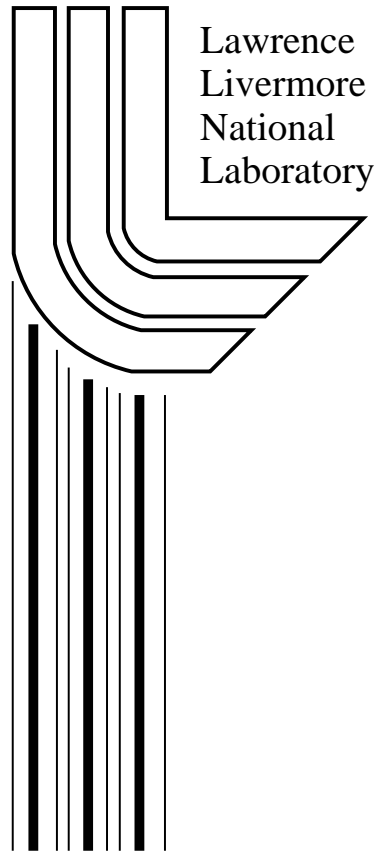

August 10, 2005 


\section{DISCLAIMER}

This document was prepared as an account of work sponsored by an agency of the United States Government. Neither the United States Government nor the University of California nor any of their employees, makes any warranty, express or implied, or assumes any legal liability or responsibility for the accuracy, completeness, or usefulness of any information, apparatus, product, or process disclosed, or represents that its use would not infringe privately owned rights. Reference herein to any specific commercial product, process, or service by trade name, trademark, manufacturer, or otherwise, does not necessarily constitute or imply its endorsement, recommendation, or favoring by the United States Government or the University of California. The views and opinions of authors expressed herein do not necessarily state or reflect those of the United States Government or the University of California, and shall not be used for advertising or product endorsement purposes.

This is a preprint of a paper intended for publication in a journal or proceedings. Since changes may be made before publication, this preprint is made available with the understanding that it will not be cited or reproduced without the permission of the author.

This research was supported under the auspices of the U.S. Department of Energy by the University of California, Lawrence Livermore National Laboratory under contract No. W-7405Eng-48. 


\title{
Numerical Algorithms for Two-Dimensional Dry Granular Flow with Deformable Elastic Grains
}

\author{
Henry A. Boateng \\ Department of Mathematics \\ University of Michigan \\ Valjean Elander \\ Department of Mathematics \\ University of Nevada, Las Vegas \\ Chao Jin \\ Department of Mathematics \\ University of Colorado, Boulder \\ Yan Li \\ Department of Mathematics \\ Texas A\& $M$ University \\ Paula Vasquez \\ Department of Mathematics \\ University of Delaware \\ Petri Fast (mentor) \\ Center for Applied Scientific Computing \\ Lawrence Livermore National Laboratory
}

August 10, 2005 


\section{INTRODUCTION}

We consider the dynamics of interacting elastic disks in the plane. This is an experimentally realizable two-dimensional model of dry granular flow where the stresses can be visualized using the photoelastic effect. As the elastic disks move in a vacuum, they interact through collisions with each other and with the surrounding geometry. Because of the finite propagation speed of deformations inside each grain it can be difficult to capture computationally even simple experiments involving just a few interacting grains.

The goal of this project is to improve our ability to simulate dense granular flow in complex geometry. We begin this process by reviewing some past work, how we can improve upon previous work. The focus of this project is on capturing the elastic dynamics of each grain in an approximate, computationally tractable, model that can be coupled to a molecular dynamics scheme.

This project was started by researching the basic molecular dynamics(MD) algorithm[5], described by Rapaport[5], which simply integrates Newton's second law and models grain interactions through a Lennard-Jones (LJ) potential. The LJ potential was originally proposed for liquid argon to calculate the potential energy between a pair of atoms $i$ and $j$ located at $\mathbf{r}_{\mathbf{i}}$ and $\mathbf{r}_{\mathbf{j}}$. This introduces us to the concept of soft-particle MD simulation of granular flow: The particles are allowed to overlap (slightly), and particle interactions result from pairwise interparticle forces that are generated in particle overlap regions.

Classical MD methods have been developed for computing thermodynamic properties of atoms in free-space. Therefore, Rapaport [5] spends considerable time on discussions of periodic boundary conditions, which is the traditional approach for concentrating computational effort in a finite region without boundary effects. This means that he considers the particles to be in a domain of infinite space. For MD simulation of granular flow, this may be a good beginning idea to consider, but clearly boundary effects will be important in our problem.

Soft-disk fluid particles were considered initially, but with fixed, rigid, non-periodic boundaries conditions. In the case of solid grains, periodic boundary conditions aren't realistic. A physical example of this is the case of studying the force chains created by granular particles, such as rice, in a silo. The particles would collide with the boundary and there would be a resulting force. The equations used by Rapaport that define the simulation algorithm were derived based on empirical results. We want to avoid this by finding an algorithm whose constants have physical meaning, and we want our results to predict and model the results from an experiment without using the results from the experiment to begin with.

Cundall and Strack[2] used a distinct element method as a tool for research into the behavior of granular assemblies. They also derived force laws using Newton's second law applied to a disk. The equations derived in this paper are equivalent to equations derived in the Ciamarra paper, so we compared the two papers and analyzed the equations to explain and use the similarities between them to model a force law.

Ciamarra et. al.[1] also performed experiments that were compared with soft-disk MD simulations of two dimensional projectile impact in a model granular material. Their computational framework is a simplified version Cundall \& Strack's approach[2]. In this paper, the normal and tangential forces were also calculated pairwise for all the particles: The normal forces result from a Hertzian contact mechanics analysis, and the tangential stress models a simple friction law. How- 
ever, as in the Rapaport simulations, the constants used in the algorithm were used as fitting parameters to match experimental observations. Once again, these simulations do not predict the experiments based on an a priori choice of parameters. Rather, the simulations match the experiments but only using an empirical a posteriori parameter fit.

As part of our project, we used our simulation to numerically model the Howell \& Behringer[3] two-dimensional granular Couette flow experiment. This consists of again comparing forces between each pair of interacting particles, mearsuing them, and calculating their new locations and forces at each time step. The annular domain was modeled by ghost particles which line the boundaries of the physical domain and contain grains inside the domain by imparting a repulsive force them.

Finally, the models were implemented for simulation studies. Using MATLAB, we wrote a routine to gather the input parameters and to create the domain to use for the simulation. We used the two domains, a square and an annulus. Within this routine, both boundary points and interior points are created. All of this data is then written to a text file that is read in by a program written in $\mathrm{C}$ that performs the more challenging computations of computing the forces between particles and determining the particle positions, potentials, and forces at each time step. Considering all $n$ particles within the domain and finding the distances between each particle in order to determine if there will be an acting force on the particle requires $O\left(n^{2}\right)$ computation. To help boost computational efficiency of our MD algorithm, we made use of the linked cell method reviewed by Muth et. al.[4]. The computation efficiency and saving will be discussed in the technical section.

In addition to our numerical tribulations, you will find in section three the beginning research done in order to add elasticity to the models mentioned above. First, we must consider stresses inside a single elastic disk. After the simple case is done, we proceed to build the theory needed in order to find the stress distribution of a larger system of disks. Then we will be able to describe the interactions between each disk in a system. We start by deriving a force law pertaining to quasi-static disks before proceeding to the more complicated problem involving full freedom of movement. This will allow us to form a quasi-static model for the flow of granular elastic disks. We proceed by fist reviewing the equations of linear elasticity. These equations can be reduced to a biharmonic problem, and they can be simplified to the plane stress problem as well. Then, we construct the solution for the stress field in the disks by first solving the problem in the single disk with boundary conditions given by determing either stresses or displacements. Finally we consider quasistatic elastic interactions of a closely packed system of three interacting disks. This points future possibilities with a large number of interacting closely packed elastic disks. 


\section{MOLECULAR DYNAMICS}

\subsection{Description}

Molecular dynamics simulation numerically integrates Newton's equations of motion to compute the position of the particles in a molecular system and obtain information about their time-dependent properties and interactions. A simulation involves calculating the forces acting on each particle at every time step. The physics is specified by the choice of the force law. One computational wall is the choice of time step. We will discuss this problem more in the technical section. Note that when two particles are very close together, it creates a very large force. So, if there are two particles moving towards each other and the time step is too large, the new positions of the two particles might end up being almost fully overlapping. This will create forces going to infinity. Such time steps, though, are efficient because they mean fewer computations. To prevent forces of excessively high numbers, it is necessary to decrease the time step. This reduction, however, causes poor computational efficiency.

The motion of each disk is obtained by integrating Newton's equations with the forces and torques produced by its interaction with all neighboring disks and the boundary. One flexibility of these simulations is the choice of force calculation method. Another flexibility comes from the type of numerical integration used. We considered the leapfrog (or Verlet) method for our integration.

Assumptions we made in all of our simulations were that our particles are non-cohesive, dry, and inelastic. We began by revisiting the work of [5], and used a Lennard-Jones potential to calculate the forces using soft-disk particles. This model accounts for repulsion and attraction of the particles within a short-range of each other. Using this potential-driven force law, we tested both rotating and non-rotating particles within a fixed, rigid boundary, i.e. the 'ghost' boundary particles did not move or rotate. Next, we used a force law combining shear and normal forces based on the technique developed in [1]. It incorporates an elastic-plastic interaction as well as static friction. The shear force is proportional to the integrated tangential displacement and is limited by the product of the static friction coefficient and the instantaneous normal force.

\section{$2.2 \quad$ Force Laws}

In this part, we will consider different models for normal and tangential forces. Newton's second law is then employed to compute normal and tangential accelations. Before considering each model, we give the Newton's formulas:

\section{Newton's second law}

$$
\begin{aligned}
& m_{i} \ddot{\mathbf{r}}_{i j}=\sum_{j=1, j \neq i}^{N} \mathbf{f}_{i j}^{n} \\
& I_{i} \ddot{\theta}_{i}=\sum_{j=1, j \neq i}^{N} \mathbf{f}_{i j}^{s}
\end{aligned}
$$

where, 
$\mathbf{r}_{i j}=\mathbf{r}_{i}-\mathbf{r}_{j}$ is the relative position of disk $i$ to disk $j$;

$\theta_{i}$ is the rotating angle of disk $i$;

$m_{i}$ is the mass of disk $i$;

$I_{i}=\frac{1}{2} m_{i} R_{i}^{2}$ is the moment of inertia of disk $i$;

$\mathbf{f}_{i j}^{n}$ is the normal force that disk $j$ exerts on disk $i$;

$\mathbf{f}_{i j}^{s}$ is the tangential force that disk $j$ exerts on disk $i$;

By the above law, we can see the sum is over all discs, excluding $i$ itself. By Newton's third law, each disk pair need only be examined once.

Newton's third Law

$$
\mathbf{f}_{\mathrm{ij}}=-\mathbf{f}_{\mathrm{ji}}
$$

Once we have normal and tangential accelarations, we apply the following leapfrog integration equations to each component of each disk's coordinates and velocities.

$$
\begin{aligned}
& \dot{\mathbf{r}}_{i}\left(t+\frac{\triangle t}{2}\right)=\dot{\mathbf{r}}_{i}\left(t-\frac{\triangle t}{2}\right)+\ddot{\mathbf{r}}_{i}(t) \triangle t \\
& \mathbf{r}_{i}(t+\triangle t)=\mathbf{r}_{i}(t)+\dot{\mathbf{r}}_{i}\left(t+\frac{\triangle t}{2}\right) \triangle t \\
& \dot{\theta}_{i}\left(t+\frac{\triangle t}{2}\right)=\dot{\theta}_{i}\left(t-\frac{\triangle t}{2}\right)+\ddot{\theta}_{i}(t) \triangle t \\
& \theta_{i}(t+\triangle t)=\theta_{i}(t)+\dot{\theta}_{i}\left(t+\frac{\triangle t}{2}\right) \triangle t
\end{aligned}
$$

\subsubsection{Lennard-Jones Model}

We study normal forces between disks using a Lennard-Jones potential. Using this model, we will get the repulsion and attraction forces between pair of discs.

For a pair of discs $i$ and $j$ located at $\mathbf{r}_{i}$ and $\mathbf{r}_{j}$, the potential formula is:

$$
U\left(r_{i j}\right)=4 \epsilon\left[\left(\frac{\sigma}{r_{i j}}\right)^{12}-\left(\frac{\sigma}{r_{i j}}\right)^{6}\right] \quad r_{i j} \leq r_{c}
$$

where $\mathbf{r}_{i j}=\mathbf{r}_{i}-\mathbf{r}_{j}$, and $r \equiv|\mathbf{r}|$. The parameter $\epsilon$ governs the strength of the interaction, and $\sigma$ defines a length scale. The interaction repels at close range, but then there is no force when they are separated by a distance greater than a cutoff distance, $r_{c}$.

The force corresponding to $U(r)$ is $\mathbf{f}=-\nabla U(r)$, so the force that disk $j$ exerts on disk $i$ is

$$
\mathbf{f}_{i j}=\frac{48 \epsilon}{\sigma^{2}}\left[\left(\frac{\sigma}{r_{i j}}\right)^{14}-0.5\left(\frac{\sigma}{r_{i j}}\right)^{8}\right] \mathbf{r}_{i j} \quad r_{i j} \leq r_{c} .
$$


Now, we can apply Newton's second law to get accelation by applying the formula

$$
m_{i} \ddot{\mathbf{r}}_{i j}=\mathbf{F}_{i}=\sum_{j=1, j \neq i}^{N_{a}} \mathbf{f}_{i j} .
$$

\subsubsection{Ciamarra Model}

Ciamarra et. al.[1] gave the following model for a granular medium. They use a heaviside function in shear force, which models an elastic-plastic interaction. The use of the heaviside function distinguishes it from other soft-disk MD simulations. This model has a simple form and it is very realistic and computationally efficient.

The normal and tangential forces between disk $i$ with radius $R_{i}$ and disk $j$ with radius $R_{j}$ are given by

$$
\begin{aligned}
& \mathbf{F}_{i j}^{n}=\left[k\left(R_{i}+R_{j}-r_{i j}\right)+m_{r_{i j}} \gamma_{n}\left|\mathbf{v}_{i}^{n}\right| \delta\left(\mathbf{v}_{i}^{n}\right)\right] \mathbf{n}_{i j} \\
& \mathbf{F}_{i j}^{s}=\min \left(m_{r_{i j}} \gamma_{s}\left|\mathbf{v}_{i}^{s}\right|, \mu\left|\mathbf{F}_{i j}^{n}\right|\right) \mathbf{s}_{i j},
\end{aligned}
$$

where the unit vector $\mathbf{n}_{i j}$ is pointing from the center of disc $j$ to the center of disk $i$. This vector is given by $\mathbf{n}_{i}=\frac{\mathbf{r}_{i j}}{r_{i j}}$. The unit vector $\mathbf{s}_{i j}$ is obtained by a clockwise rotation of $\mathbf{n}_{i j}$ through $\frac{\pi}{2}$.

The normal and the tangential components of the surface velocity are given, repectively, by

$$
\begin{gathered}
\mathbf{v}_{i}^{n}=\mathbf{v}_{i} \cdot \mathbf{n}_{i j}, \\
\mathbf{v}_{i}^{s}=\mathbf{v}_{i} \cdot \mathbf{s}_{i j}+\dot{\theta}_{i} R_{i} .
\end{gathered}
$$

The reduced mass is $m_{r_{i j}}^{-1}=m_{i}^{-1}+m_{j}^{-1}$. A heaviside function, $\delta(\cdot)$, models an elastic plastic interaction.

The viscoelastic constants, $k=3.2 \times 10^{3} \mathrm{kgs}^{-2}, \gamma_{n}=10^{4} \mathrm{~s}^{-1}$, and $\gamma_{s}=8 \times 10^{3} \mathrm{~s}^{-1}$ and the static friction coefficient, $\mu=0.28$, are found empirically in [1].

\subsubsection{The Cundall and Strack Model}

Cundall and Strack[2] developed a discrete element method that incorporates a force-displacement law, Coulomb-type friction law, and Newton's second law.

The relative velocity of the disks at their contact point is

$$
\mathbf{V}_{i j}=\left(\mathbf{v}_{i}-\mathbf{v}_{j}\right)-\left(\dot{\theta}_{i} R_{i}+\dot{\theta}_{j} R_{j}\right) \mathbf{n}_{i j} .
$$


The normal $\dot{\mathbf{r}}_{i j}$ and tangential $\dot{\theta}_{i j}$ components of the relative velocities are the projections of $\mathbf{V}_{i j}$ onto $\mathbf{n}_{i j}$ and $\mathbf{s}_{i j}$, respectively, where

$$
\begin{aligned}
& \dot{\mathbf{r}}_{i j}=\mathbf{V}_{i j} \mathbf{n}_{i j}=\left(\mathbf{v}_{i}-\mathbf{v}_{j}\right) \mathbf{n}_{i j}-\left(\dot{\theta}_{i} R_{i}+\dot{\theta}_{j} R_{j}\right) \mathbf{n}_{i j} \mathbf{s}_{i j}=\left(\mathbf{v}_{i}-\mathbf{v}_{j}\right) \mathbf{n}_{i j} \\
& \dot{\theta}_{i j}=\mathbf{V}_{i j} \mathbf{s}_{i j}=\left(\mathbf{v}_{i}-\mathbf{v}_{j}\right) \mathbf{s}_{i j}-\left(\dot{\theta}_{i} R_{i}+\dot{\theta}_{j} R_{j}\right) \mathbf{s}_{i j} \mathbf{s}_{i j}=\left(\mathbf{v}_{i}-\mathbf{v}_{j}\right) \mathbf{s}_{i j}-\left(\dot{\theta}_{i} R_{i}+\dot{\theta}_{j} R_{j}\right) .
\end{aligned}
$$

Integration of relative displacement increments for position and angle are

$$
\begin{aligned}
& \triangle \mathbf{r}_{i j}=\dot{\mathbf{r}}_{i j} \triangle t=\left[\left(\mathbf{v}_{i}-\mathbf{v}_{j}\right) \mathbf{n}_{i j}\right] \triangle t \\
& \triangle \theta_{i j}=\dot{\theta}_{i j} \triangle t=\left[\left(\mathbf{v}_{i}-\mathbf{v}_{j}\right) \mathbf{s}_{i j}-\left(\dot{\theta}_{i} R_{i}+\dot{\theta}_{j} R_{j}\right)\right] \triangle t .
\end{aligned}
$$

The relative displacement increments are used with the following Force-Displacement Law to calculate increments of the normal and tangential forces

$$
\begin{aligned}
& \triangle \mathbf{F}_{i j}^{n}=\tilde{k^{n}} \triangle \mathbf{r}_{i j}=\tilde{k^{n}}\left[\left(\mathbf{v}_{i}-\mathbf{v}_{j}\right) \mathbf{n}_{i j}\right] \triangle t \\
& \triangle \mathbf{F}_{i j}^{s}=\tilde{k^{s}} \triangle \theta_{i j}=\tilde{k^{s}}\left[\left(\mathbf{v}_{i}-\mathbf{v}_{j}\right) \mathbf{s}_{i j}-\left(\dot{\theta}_{i} R_{i}+\dot{\theta}_{j} R_{j}\right)\right] \triangle t,
\end{aligned}
$$

where, $\tilde{k^{n}}$ and $\tilde{k^{s}}$ are normal and tangential stiffness.

Finally, at each time step $t=t_{N}$ the force increments $\triangle \mathbf{F}_{i j}^{n}$ and $\triangle \mathbf{F}_{i j}^{s}$ are added into the sum of all force increments. And then $F_{i j}^{n}$ and $F_{i j}^{s}$ are determined for previous time steps

$$
\left(\mathbf{F}_{i j}^{n}\right)_{N}=\left(\mathbf{F}_{i j}^{n}\right)_{N-1}+\triangle \mathbf{F}_{i j}^{n} ;\left(\mathbf{F}_{i j}^{s}\right)_{N}=\left(\mathbf{F}_{i j}^{s}\right)_{N-1}+\triangle \mathbf{F}_{i j}^{s}
$$

The Coulomb-type Friction Law is incorporated as follows:

The magnitude of the shear force $F^{s}$ is checked against the maximum possible value $\left(F^{s}\right)_{\max }$, defined as:

$$
\left(\mathbf{F}^{s}\right)_{\max }=\left|\tan \left(\phi_{\mu}\right) \cdot \mathbf{F}^{n}+c\right|,
$$

where $\phi_{\mu}$ is the smaller of the interparticle friction angles of the two discs in contact and $c$ is the smaller of their cohesions.

At last, we derive the forces formula

$$
\begin{aligned}
& \left(\mathbf{F}_{i j}^{n}\right)_{N}=\left(\mathbf{F}_{i j}^{n}\right)_{N-1}+\triangle \mathbf{F}_{i j}^{n} \\
& \left(\mathbf{F}_{i j}^{s}\right)_{N}=\min \left[\left(\mathbf{F}_{i j}^{s}\right)_{N-1}+\triangle \mathbf{F}_{i j}^{s},\left(\left(\mathbf{F}_{i j}^{s}\right)_{\max }\right)_{N}\right] .
\end{aligned}
$$

\section{$2.3 \quad$ Numerical Implementation}

In the implementation, we simulate Lennard-Jones force and Ciamarra force models on different geometric domains. The Lennard-Jones force omitted the circular movements of the molecules. The Ciamarra force takes this into consideration. 
We iterate the simulation in increasing time steps. At each time step, we compute the new positions, forces, velocities, and accelerations of the molecules by use of the Leapfrog numerical method, and output these variables to create graphics. To make the simulation more efficient and capable, we use $\mathrm{C}$ to code the heavy computation part and leave the graphing to Matlab.

\subsubsection{Description}

Our implementation of the molecular dynamics (MD) method is outlined in Algorithm 1. We follow the basic ideas discussed in Rapaport [5]. When calculating the force between the molecules, we assume that if the distance is greater than some cutoff, denoted as $r_{c}$, there is no force between them. If we calculate the distance and forces for all the pairs of the molecules, the computation is about $O\left(2 n^{2}\right)$, where $n$ is the number of the molecules. This is a lot of work if we simulate many molecules.

To decrease the computation work, we cut the domain of the movement into small boxes. We assume that only the molecules in the same box and the neighboring boxes interact. A list records which molecules are in each box. For each iteration, we update the lists. The computation is about $O(n)$. Next, we compute the forces between the molecules. The computation is about $O\left(c n^{2}\right)$, where $c<1$, depending on the number of boxes.

With the forces, we can use Newton's second law to obtain the acceleration of the molecules. Then we use Leapfrog to solve the velocity and new positions of the molecules. This finishes one iteration.

\subsubsection{Boundary Geometry}

We considered a square domain and an annulus domain. Since the forces on the interior particles depend on the boundary when it is close enough, we had to decide how to allow them to interact. One way is to think of the boundary as a straight rigid wall as [2] does. This approach adds complexity that, firstly, is not necessary for the fixed, non-moving walls we are using, and, secondly, is beyond our project time limit. We decided on the more simplistic engineering approach of putting 'ghost' particles outside of the boundary that would interact with the interior particles. In this case, if there are $i$ interior particles and $b$ boundary particles, then $n=i+b$, where $n$ is the total number of particles in the system.

In the initial setup of the geometry and the particles, all particles are of the same radius. In the case of the square domain, if the side of the square is length $l$, then we would like all the 'ghost' particles to be outside the domain, tangent to the sides. The centers of these fixed 'ghost' particles could go right on the edge of the boundary of the square, but it would somewhat complicate things by making the real square domain size $(l-2 r)^{2}$ instead of $l^{2}$, given that the particles are of radius $r$. By putting the 'ghost' particles around the boundary, it allows for interaction with the interior particles, so a force is calculated between the interior and the boundary particles. This gives the force and causes the interior particles to repel away from the boundary. One thing to consider in generating the particles on the boundary is overlap. There are three cases of overlapping: 


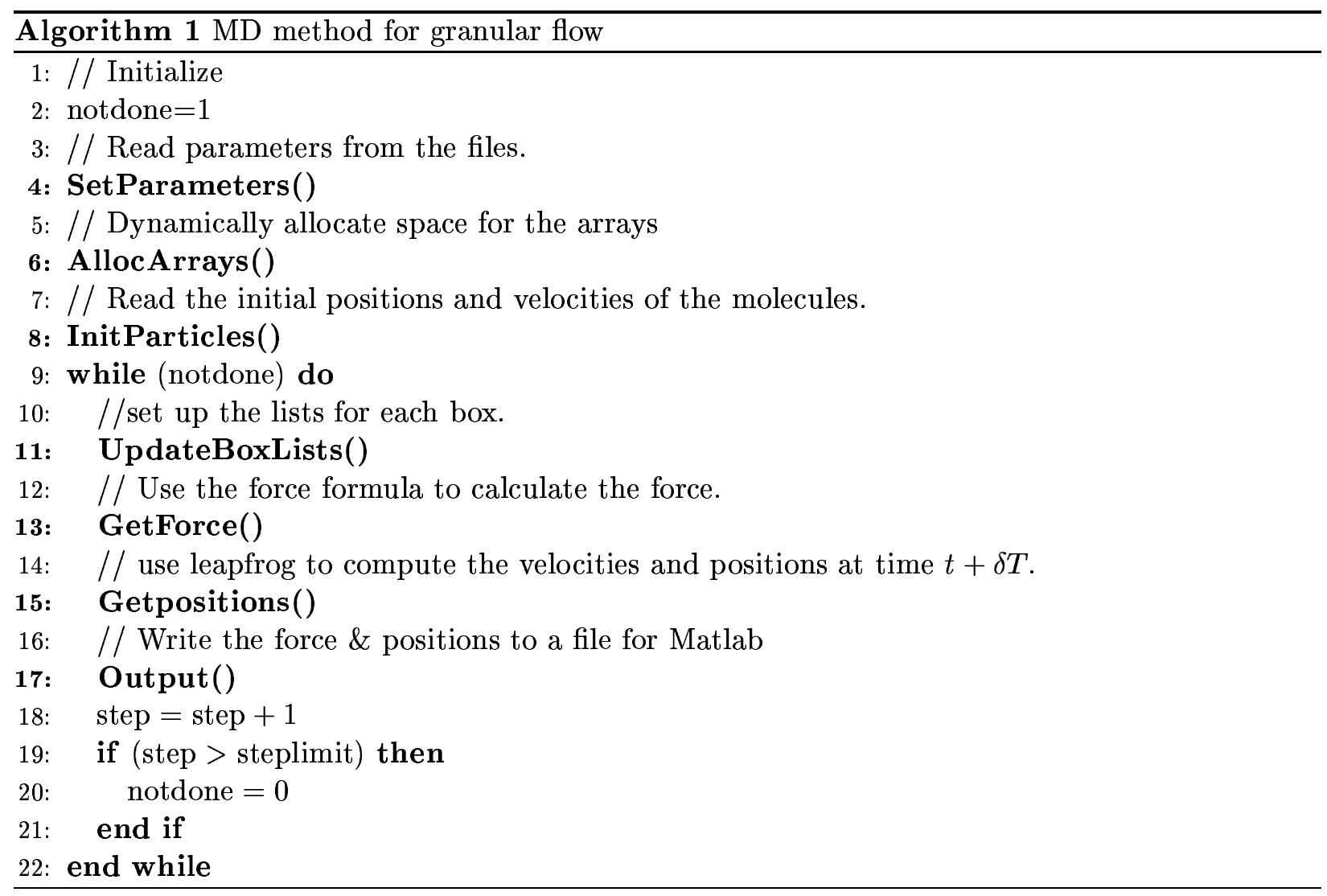

1. There are gaps between the boundary particles.

2. There are no gaps between the boundary particles, but they do not overlap, either. They are tangential to each other.

3. The boundary particles are overlapping.

In the first case, one needs to calculate how far away from each other the particles need to be. If they are too far away from each other, then a force might not be created that is strong enough to repel the interior particles. This would cause the interior particles to escape the domain. So, one must consider that the more space there is between each boundary particle, the less force will be exerted on an interior particle that comes very close to that location. In the second case, there is no possibility of escaping particles. This is what we used to begin with in our testing. And, in the third case, there is no possibility of escaping particles; however, in this case, the force acting on the interior particle that comes very close will be greater, and this should repel the inner particle away from that area more quickly. Given large time steps, if a particle is allowed to get too close to these overlapping boundary particles, one will find the programming generating forces of extremely high magnitude. In conclusion, by changing the number of 'ghost' boundary particles, one can possibly change the elasticity of the boundary. More research should be done to compare how the density of the boundary particles is related to the elasticity of the boundary media. We tested all three of the above cases. Some results are shown later. 
The second domain considered was an annulus. The physical representation of this domain is that of a 2-D granular Couette flow[3]. Our motivation for simulating this domain is so that we can compare our results with the experimental results [3] obtains. First, we studied the case where both the interior and exterior walls were fixed and non-moving. Then, we allowed the inner boundary to rotate at some constant velocity. Again, overlap of the boundary particles could pose some interesting research topics here.

\subsubsection{Stability Analysis}

We use the leapfrog scheme as our integrator to update the velocity and positions of our forces. We will try to establish a rough upper bound for our timestep $\delta t$, for a simplified case of our problem, that ensures that the leapfrog scheme is absolutely stable.

- The Scheme The general leapfrog difference scheme is

$$
\frac{U_{n+1}-U_{n-1}}{2 \delta t}=f\left(U_{n}\right)
$$

For the test equation $y^{\prime}=\lambda y$, the difference scheme is

$$
U_{n+1}-U_{n-1}=2 \delta t \lambda U_{n}
$$

The characteristic equation for the leapfrog method is

$$
\zeta^{2}-2 \delta t \lambda \zeta-1=0
$$

where $\zeta_{1}$ and $\zeta_{2}$ are the roots of the characteristic equation. Therefore, $U_{n}=c_{1} \zeta_{1}^{n}+c_{2} \zeta_{2}^{n}$ where $c_{1}$ and $c_{2}$ are constants and

$$
\begin{aligned}
& \zeta_{1}=\delta t \lambda+\left[1+(\delta t \lambda)^{2}\right]^{\frac{1}{2}} \\
& \zeta_{2}=\delta t \lambda-\left[1+(\delta t \lambda)^{2}\right]^{\frac{1}{2}} .
\end{aligned}
$$

The scheme is absolutely stable if $\left|\zeta_{i}\right| \leq 1$, for $i=1,2$. And, if $\left|\zeta_{i}\right|=1$, then $\zeta_{i}$ has to be a simple root.

\section{- Taylor Expansion}

Expanding each root, $\zeta_{1}$ and $\zeta_{2}$, given above, yields the equivalencies

$$
\begin{aligned}
& \zeta_{1}=1+\delta t \lambda+\frac{(\delta t \lambda)^{2}}{2}+O\left(\delta t^{3}\right)=e^{\delta t \lambda}+O\left(\delta t^{3}\right) \\
& \zeta_{2}=-1+\delta t \lambda-\frac{(\delta t \lambda)^{2}}{2}+O\left(\delta t^{3}\right)=-e^{-\delta t \lambda}+O\left(\delta t^{3}\right)
\end{aligned}
$$

Dropping the $O\left(\delta t^{3}\right)$ term and setting $\left|\zeta_{i}\right|=1$ implies that $\lambda$ is purely imaginary. 
A short proof of this is

$$
\begin{aligned}
& \zeta=\delta t \lambda \pm\left[1+(\delta t \lambda)^{2}\right]^{\frac{1}{2}} \\
& \zeta^{2}=1+2(\delta t \lambda)^{2} \pm 2 \delta t \lambda\left[1+(\delta t \lambda)^{2}\right]^{\frac{1}{2}} \\
& \zeta^{2}=1+2(\delta t \lambda)\left[\delta t \lambda \pm\left[1+(\delta t \lambda)^{2}\right]^{\frac{1}{2}}\right] \\
& \zeta^{2}=1+2(\delta t \lambda \zeta)
\end{aligned}
$$

This gives the function

$$
\delta t \lambda(\zeta)=\frac{1}{2}\left(\zeta-\frac{1}{\zeta}\right)
$$

If $\zeta=r e^{i \theta}$, then $\delta t \lambda(\zeta)=\frac{1}{2}\left[\left(r-\frac{1}{r}\right) \cos \theta+i\left(r+\frac{1}{r}\right) \sin \theta\right]$. So, by setting $|\zeta|=1$, we get both that $r=1$ and $\delta t \lambda(\zeta)=i \sin \theta$. Therefore, $\delta t \lambda$ lies strictly between $-i$ and $i$ on the imaginary axis.

Since $\zeta_{1}$ and $\zeta_{2}$ are the roots of Eq. $(26)$, then $\left(\zeta-\zeta_{1}\right)\left(\zeta-\zeta_{2}\right)=0$, which is the same as

$$
\zeta^{2}-\left(\zeta_{1}+\zeta_{2}\right) \zeta+\zeta_{1} \zeta_{2}=0
$$

If we compare Eq.(27) with Eq.(26), we will see that $\zeta_{1} \zeta_{2}$ must be -1 . Hence, if both roots do not lie on the circle, then one lies inside the circle and the other outside the circle, making the scheme unstable. Thus, both roots must lie on the circle. This also implies that $\delta t \lambda$ lies between $i$ and $-i$, and $\lambda$ then is purely imaginary.

Thus,

$$
0<|\delta t \lambda|<1
$$

implies

$$
0<\delta t<\frac{1}{|\lambda|}
$$

- The Scheme with Lennard-Jones Potential

The force derived from the the Lennard-Jones potential is

$$
\mathbf{f}_{\mathbf{i j}}=\left(\frac{48 \epsilon}{\sigma^{2}}\right)\left[\left(\frac{\sigma}{r_{i j}}\right)^{14}-\frac{1}{2}\left(\frac{\sigma}{r_{i j}}\right)^{8}\right] \mathbf{r}_{\mathbf{i} \mathbf{j}}
$$

which is the force particle $j$ exerts on particle $i$. From Newton's second law, the total force on a particle is

$$
m \ddot{\mathbf{r}}=\sum_{j \neq i}^{N_{a}} \mathbf{f}_{\mathbf{i j}}
$$

\section{- Two Particles}

For two particles, one stationary on the $\mathrm{x}$-axis and the other moving along the $\mathrm{x}$-axis,

$$
\ddot{x}=\left(\frac{48 \epsilon}{m \sigma^{2}}\right)\left[\left(\frac{\sigma}{x}\right)^{14}-\frac{1}{2}\left(\frac{\sigma}{x}\right)^{8}\right] x
$$




$$
\ddot{x}=\left(\frac{48 \epsilon \sigma^{6}}{m}\right)\left[\frac{\sigma^{6}}{x^{6}}-\frac{1}{2 x^{7}}\right]=C \Phi(x)
$$

where $C=\frac{48 \epsilon \sigma^{6}}{m}$.

- Linearizing $\Phi(\mathrm{x})$ about $\mathrm{x}=\mathrm{d}$

By letting $\Phi(x) \approx \Phi(a)+\Phi^{\prime}(a)(x-d)$, we see that

$$
\Phi(x) \approx\left(\frac{\sigma^{6}}{d^{13}}-\frac{1}{2 d^{8}}\right)+\left(\frac{-13 \sigma^{6}}{d^{14}}+\frac{7}{2 d^{8}}\right)(x-d) .
$$

By expanding and collecting terms, we find that,

$$
\Phi(x) \approx \frac{14 \sigma^{6}}{d^{14}}-\frac{4}{d^{7}}+\left(\frac{-13 \sigma^{6}}{d^{14}}+\frac{7}{2 d^{8}}\right) x .
$$

Dropping the constant term and setting $\ddot{x}=\omega \Phi(x)$ gives us $\omega=C\left(\frac{-13 \sigma^{6}}{d^{14}}+\frac{7}{2 d^{8}}\right)$.

Since $\mathbf{x}$ is the distance, $\mathbf{v}=\dot{\mathbf{x}}$ is the velocity, and $\ddot{x}=\dot{v}$ is the acceleration, we can write our equation $\ddot{x}=\omega \Phi(x)$ as the linear system

$$
\left(\begin{array}{l}
x \\
v
\end{array}\right)^{\prime}=\left(\begin{array}{l}
v \\
\ddot{x}
\end{array}\right)=\left(\begin{array}{cc}
0 & 1 \\
\omega & 0
\end{array}\right)\left(\begin{array}{l}
x \\
v
\end{array}\right)
$$

We compute the eigenvalues, $\lambda_{1,2}$ to be $\lambda_{1,2}= \pm \sqrt{\omega}$, where $\lambda$ is purely imaginary. If $\lambda$ is purely imaginary, then, $\omega<0$. To ensure that $\omega$ is less than zero, we need $\frac{-13 \sigma^{6}}{d^{14}}+\frac{7}{2 d^{8}}<0$. As a result, we establish that $\frac{\sigma}{d}>\left(\frac{7}{26}\right)^{\frac{1}{6}}$ for $\omega$ to be less than zero.

Since $0<\delta t<\frac{1}{|\lambda|}$, then $0<\delta t<\frac{1}{|\sqrt{w}|}$.

And, finally we approximate a bound on $\delta t$ to be

$$
0<\delta t<\frac{1}{\left|\sqrt{\left(\frac{48 \epsilon \sigma^{6}}{m}\right)\left(\frac{-13 \sigma^{6}}{d^{14}}+\frac{7}{2 d^{8}}\right)}\right|} .
$$

\subsection{Results}

We simulate the dynamics of the grains using the Lennard-Jones force field and the Ciamarra force field. 
Example 1: Our first simulation is in a square domain with only one interior molecule. We add some gravity to it. Our simulations exactly meets our expectation that it bounce up and down. The colors of the molecules show the forces from the neighboring molecules. Blue means almost no force. Red means intense force. see figure 1

Example 2: We put more molecules to test Lennard-Jones force. We can see the molecules overlap and some even could go through each other. This is caused by LennardJones force, which can't avoid this kind of case.

In the actual movement, the molecules have a circular movement. We added the circular movement into interaction between the molecules. Our next simulation will simulate this movement.

Example 3: Let's see two particles in the domain. We can see the circular movement from the bar in the molecules, see figure (3). We also simulate a lot of particles. figure (4) is one of our results. Note that in this simulation, we assumed that when two molecules overlap, they have interacts.

From the graphics, we can see that when the molecules have interacts, the molecule itself also has the circular movement. The circular movement is also the same as what we have expected from the our model. 


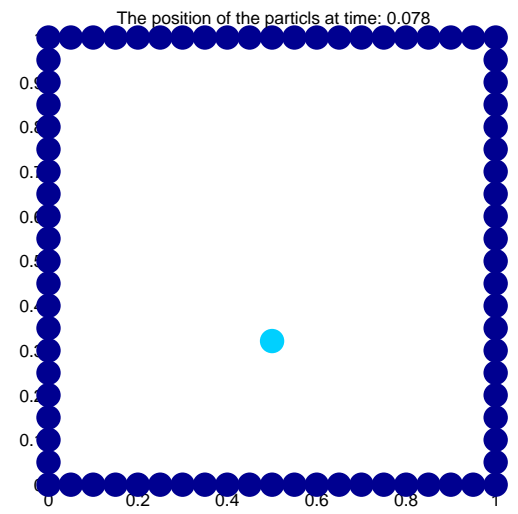

(a) $t=0.078$

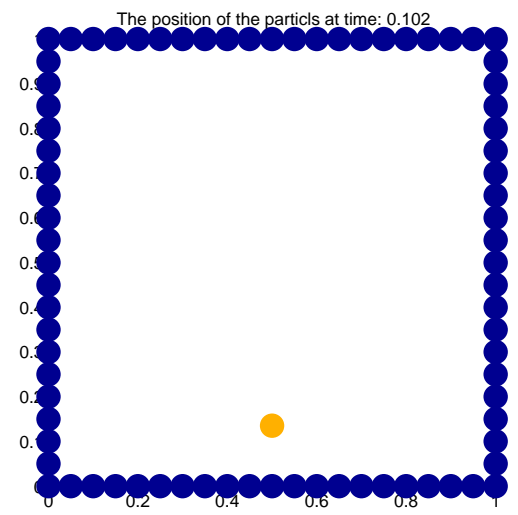

(c) $t=0.102$

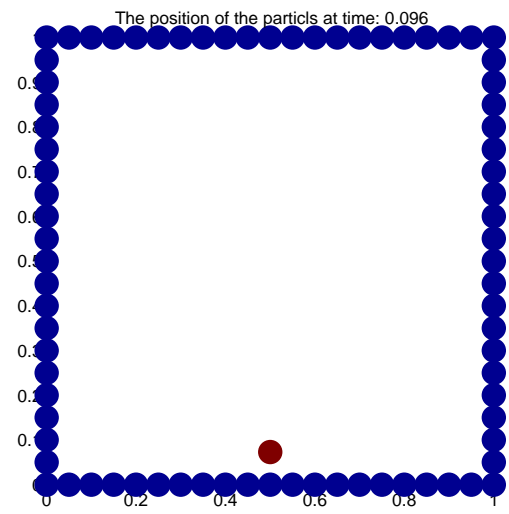

(b) $t=0.096$

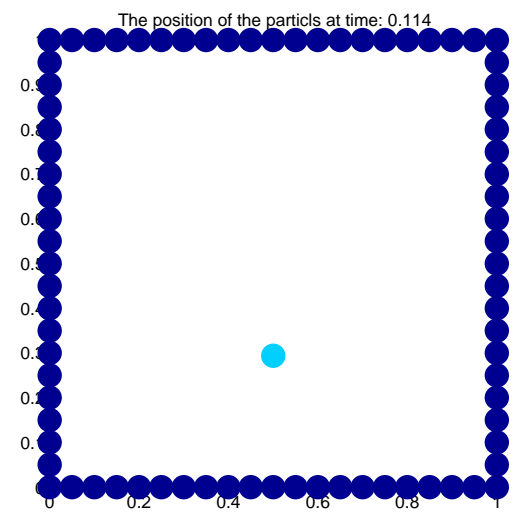

(d) $t=0.114$

Figure 1: Lennard-Jones force simulation 
The position of the particls at time: 0.006

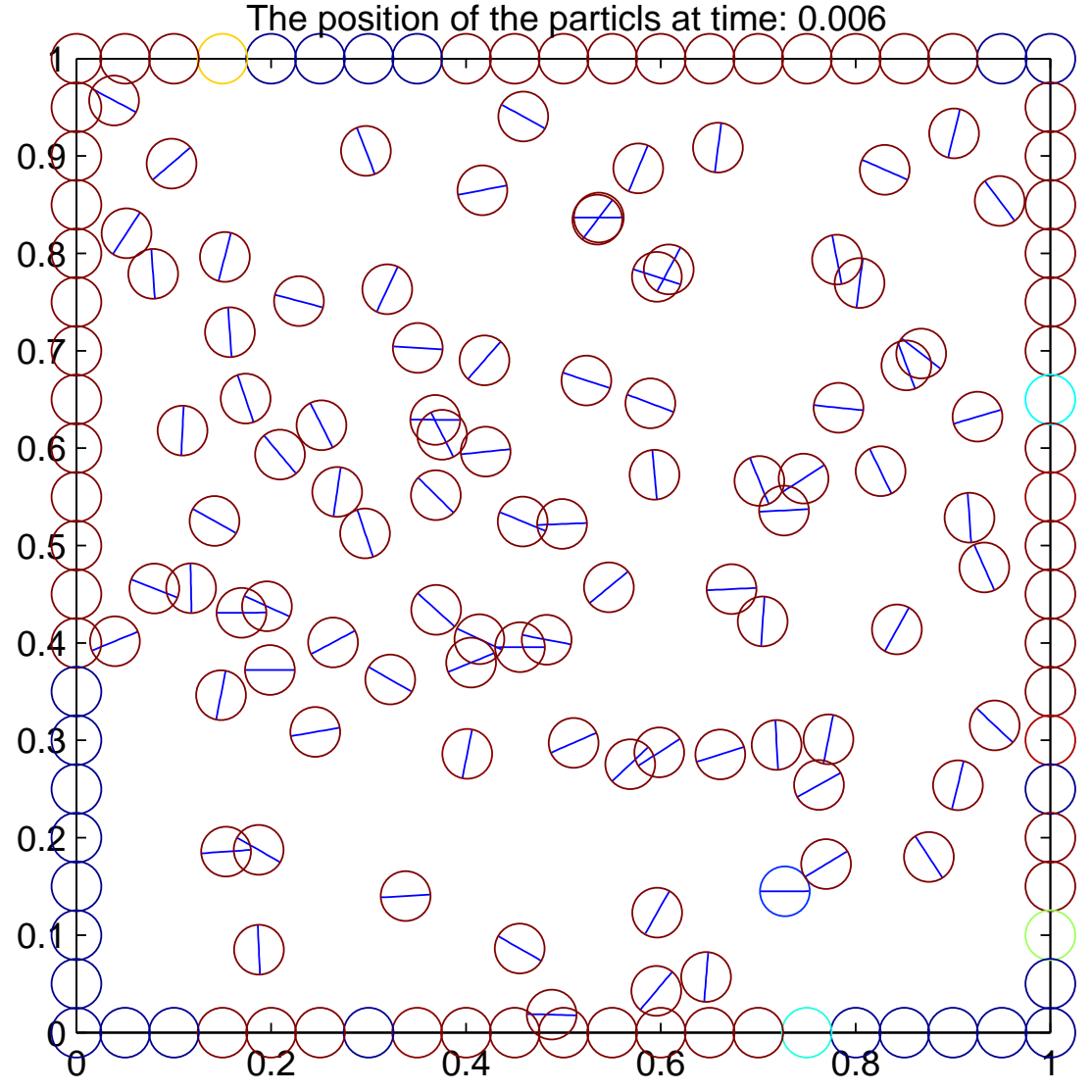

Figure 2: Lennard-Jones force simulation for many molecules 


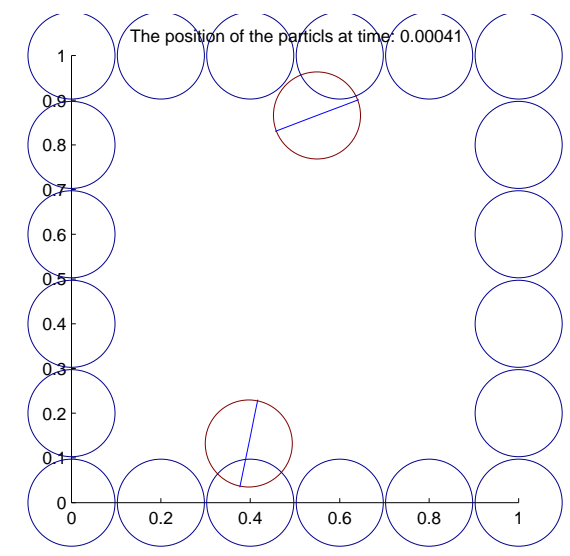

(a) $t=0.00041$

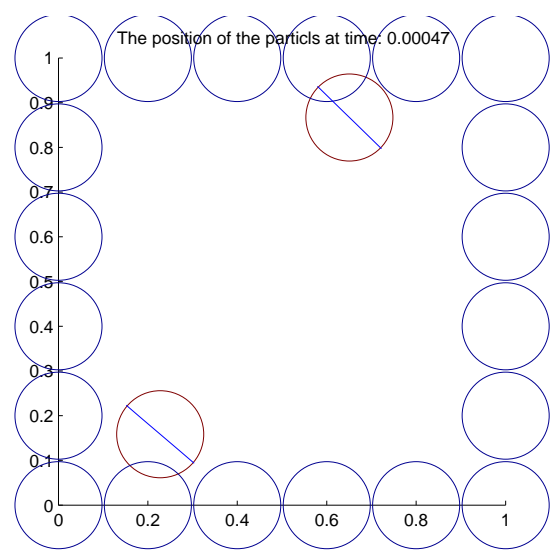

(c) $t=0.00047$

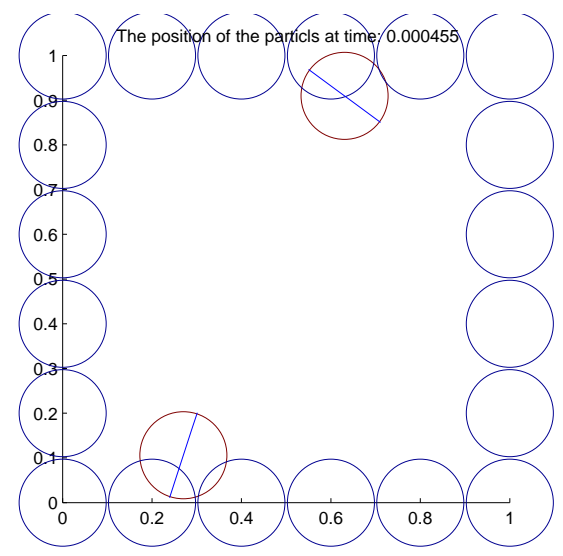

(b) $t=0.000455$

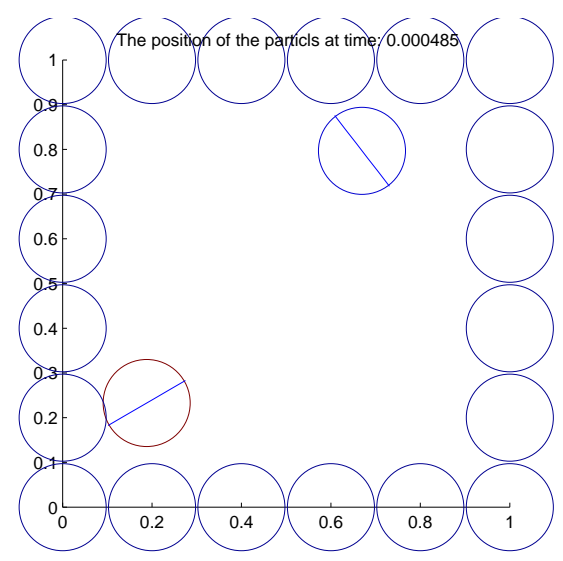

(d) $t=0.000485$

Figure 3: Ciamarra force with circular movement simulation 


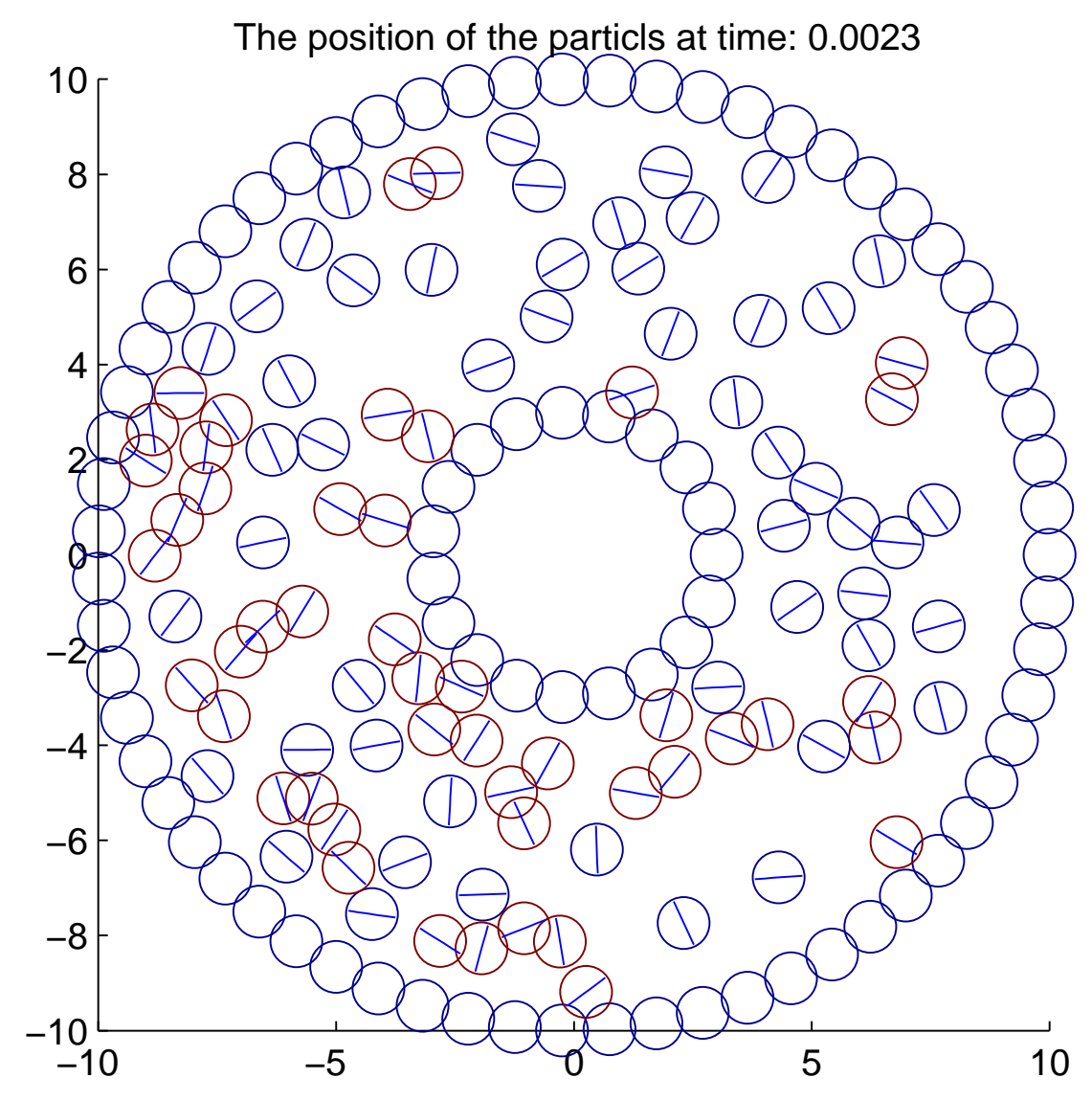

Figure 4: Ciamarra force with circular movement simulation for many molecules 


\section{ELASTICITY}

In this section the problem of finding stresses inside an elastic disk is considered. Our goal is to obtain a quasi-static description of granular flow with elastic grains derived from the stress distribution of a system of disks. We first review the equations of linear elasticity and recall how they can be simplified to the plane stress problem and subsequently to the solution of a biharmonic function. Then we construct the solution for the stress field in the disk and solve the problem in a single disk for different boundary conditions: imposed normal and tangential stresses and given displacements. Finally, we present an example of a the system with three interacting disks.

We begin with the equations of linear elasticity

- Conservation of momentum

$$
\partial_{j} T_{i j}+f_{i}=\rho \partial_{t t} u_{i}
$$

- Constitutive equation (isotropic Hooke's Law)

$$
\varepsilon_{i j}=\frac{1+\nu}{E} T_{i j}-\frac{\nu}{E} \delta_{i j} T_{k k}
$$

or

$$
T_{i j}=2 \mu \varepsilon_{i j}+(\mu+\lambda) \delta_{i j} \varepsilon_{k k}
$$

- Kinematic equation

$$
\varepsilon_{i j}=\frac{1}{2}\left(\partial_{i} u_{j}+\partial_{j} u_{i}\right)
$$

where $T_{i j}=T_{j i}$ is stress, $f_{i}$ is body force, $\rho$ is density, $u_{i}$ is displacement, $\varepsilon_{i j}=\varepsilon_{j i}$ is strain, $\lambda$ and $\mu$ are the lame constants, $E$ is the Young's modulus and, $\nu$ is the Poisson's ratio.

In the case of static plane stress,

$$
u_{1}=u_{1}\left(x_{1}, x_{2}\right), \quad u_{2}=u_{2}\left(x_{1}, x_{2}\right), \quad u_{3}=0
$$

and assuming that we can ignore body forces, the equations reduce to

$$
\nabla \cdot T=0
$$

We introduce Airy's stress function $\psi\left(x_{1}, x_{2}\right)$

$$
T_{11}=\psi_{, 22}, \quad T_{12}=-\psi_{, 12}, \quad T_{22}=\psi_{, 11}
$$

and the elastic equations can then be reduced to the biharmonic equation for $\phi$ :

$$
\nabla^{2} \nabla^{2} \psi=0
$$


To complete the mathematical problem boundary conditions are introduced, the classical approach is to specified traction on the boundary, usually decomposed into tangential and normal components. alternative we can specified displacement in the boundary.

Introducing polar coordinates we can make use of the known solution of the biharmonic equation in a disk

$\psi=a_{0}+c_{0} r^{2}+d_{1} r^{3} \sin \theta+h_{1} r^{3} \cos \theta+\sum_{n=2}^{\infty}\left[\left(a_{n} r^{n}+c_{n} r^{n+2}\right) \sin n \theta+\left(e_{n} r^{n}+g_{n} r^{n+2}\right) \cos n \theta\right]$

where the component of the stress tensor are given by

$$
\begin{aligned}
T_{r r} & =\frac{1}{r^{2}} \frac{\partial^{2} \psi}{\partial \theta^{2}}+\frac{1}{r} \frac{\partial \psi}{\partial r} \\
T_{r \theta} & =-\frac{\partial}{\partial r}\left(\frac{1}{r} \frac{\partial \psi}{\partial \theta}\right) \\
T_{\theta \theta} & =\frac{\partial^{2} \psi}{\partial r^{2}}
\end{aligned}
$$

To specified displacements in the boundary we recall that in polar coordinates the strain tensor $\varepsilon$ has the form

$$
\boldsymbol{\varepsilon}=\frac{1}{2}\left(\begin{array}{ccc}
2 \frac{\partial u_{r}}{\partial r} & \frac{1}{r} \frac{\partial u_{r}}{\partial \theta}+r \frac{\partial u_{\theta}}{\partial r} & 0 \\
\frac{1}{r} \frac{\partial u_{r}}{\partial \theta}+r \frac{\partial u_{\theta}}{\partial r} & 2 \frac{1}{r}\left(\frac{\partial u_{\theta}}{\partial \theta}+u_{r}\right) & 0 \\
0 & 0 & 0
\end{array}\right)
$$

by Hooke's law

$$
\boldsymbol{\varepsilon}=\left(\begin{array}{ccc}
T_{r r}-\nu\left(T_{\theta \theta}+T_{z z}\right) & (1+\nu) T_{r \theta} & 0 \\
(1+\nu) T_{r \theta} & T_{\theta \theta}-\nu\left(T_{r r}+T_{z z}\right) & 0 \\
0 & 0 & T_{z z}-\nu\left(T_{r r}+T_{\theta \theta}\right)
\end{array}\right)
$$

then we have

$$
\begin{aligned}
T_{z z} & =\nu\left(T_{r r}+T_{\theta \theta}\right) \\
\frac{\partial u_{r}}{\partial r} & =\frac{1}{E}\left[\left(1-\nu^{2}\right) T_{r r}-\left(\nu+\nu^{2}\right) T_{\theta \theta}\right] \\
\frac{1}{r}\left(\frac{\partial u_{\theta}}{\partial \theta}+u_{r}\right) & =\frac{1}{E}\left[\left(1-\nu^{2}\right) T_{\theta \theta}-\left(\nu+\nu^{2}\right) T_{r r}\right] \\
\frac{1}{r} \frac{\partial u_{r}}{\partial \theta}+r \frac{\partial u_{\theta}}{\partial r} & =\frac{1}{E}\left[2(1+\nu) T_{r \theta}\right]
\end{aligned}
$$

using equations (36) through (43), we find that 


$$
\begin{aligned}
E u_{r}= & -(1+\nu)(4 \nu-2) r c_{0}-(1+\nu)(4 \nu-1) r^{2} \cos (\theta) h_{1}-(1+\nu)(4 \nu-1) r^{2} \sin (\theta) d_{1} \\
& -(1+\nu) \sum_{n=2}^{\infty}\left[\left(n r^{n-1} a_{n}+(4 \nu+n-2) r^{n+1} c_{n}\right) \sin (n \theta)\right. \\
& \left.+\left(n r^{n-1} e_{n}+(4 \nu+n-2) r^{n+1} g_{n}\right) \cos (n \theta)\right] \\
E u_{\theta}= & -(1+\nu)(4 \nu-5) r^{2} \sin (\theta) h_{1}+(1+\nu)(4 \nu-5) r^{2} \cos (\theta) d_{1} \\
& -(1+\nu) \sum_{n=2}^{\infty}\left[\left(n r^{n-1} a_{n}-(4 \nu-n-4) r^{n+1} c_{n}\right) \cos (n \theta)\right. \\
& \left.-\left(n r^{n-1} e_{n}+(4 \nu-n-4) r^{n+1} g_{n}\right) \sin (n \theta)\right]
\end{aligned}
$$

We first consider the case where traction is specified on the boundary by assuming that a given normal force of the form

$$
F=A \exp ^{-w *(\theta-\alpha)^{2}}
$$

is applied at $(1, \alpha)$ and there are not shear forces on the boundary. Hence our boundary conditions are

$$
\begin{aligned}
& T_{r r}(1, \theta)=F \\
& T_{r \theta}(1, \theta)=0
\end{aligned}
$$

In the case where the displacements are given on the boundary, we also assume a gaussian form and the boundary conditions are

$$
\begin{array}{r}
u_{r}(1, \theta)=F \\
u_{r \theta}(1, \theta)=0
\end{array}
$$

We plot the norma stress difference $\left(T_{r r}-T_{\theta \theta}\right)$ in the case of a single disk in figure (5), note that the boundary condition here are: a normal force at $(1, \pi / 2)$ and zero shear stresses in $(1, \theta), 0 \leq \theta \leq 2 \pi$.

As an illustrative example for a system of three disk we plot both the normal stress difference and shear stress $\left(T_{r \theta}\right)$ in figure (6) and (7). The boundary conditions imposed are normal force from outside the system and displacements on the contact points.

The next step is to derive the force law from this disks results that can be used in modeling the flow of granular elastic materials. This time we have not completed this step. 


\section{Normal Stress Difference $\left(T_{r r}-T_{\theta \theta}\right)$}

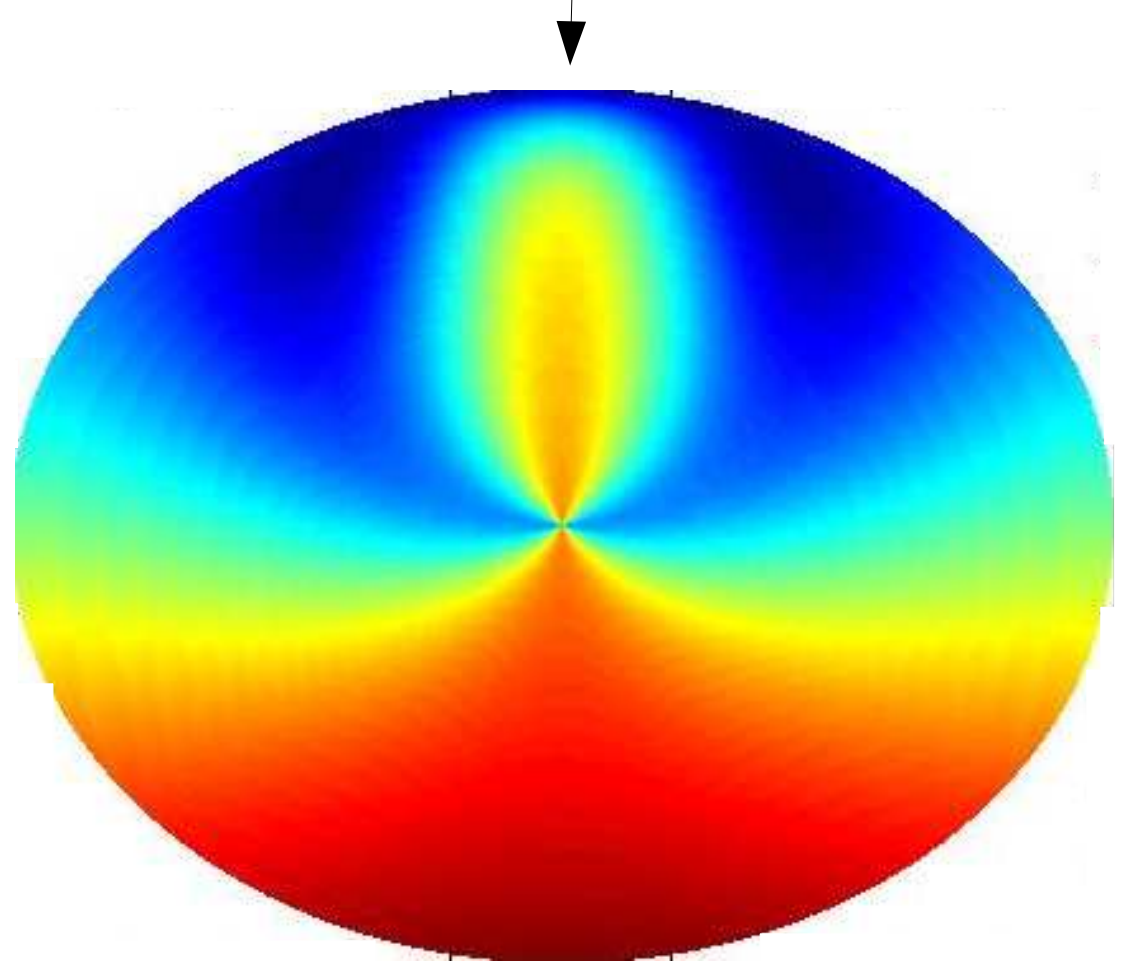

Figure 5: Normal stress distribution singular disk. Traction is specified on the boundary at $(1, \pi / 2)$ 


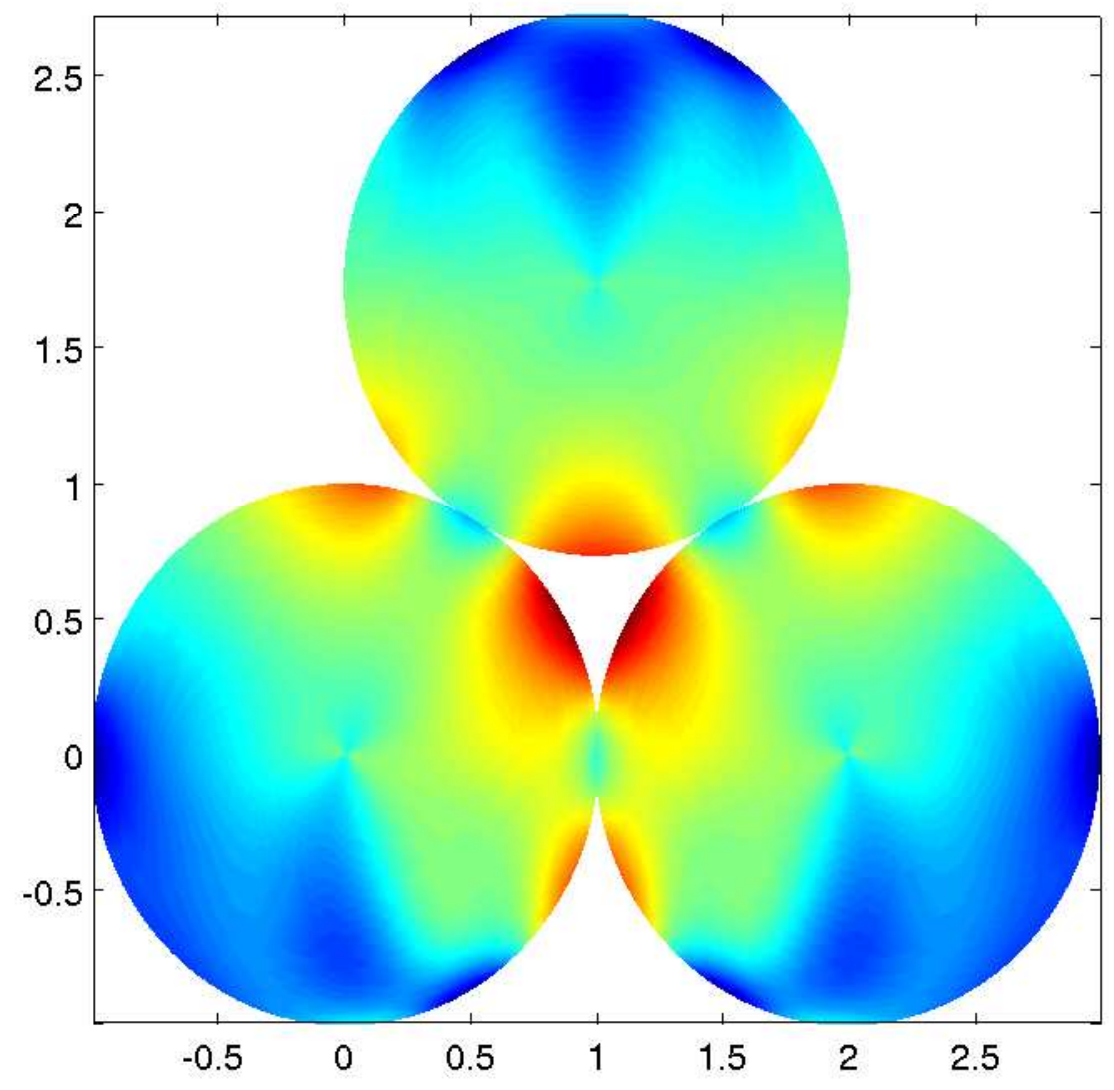

Figure 6: Normal stress distribution three disks. Traction is specified on the boundary of the system and displacement are given at the contact points 


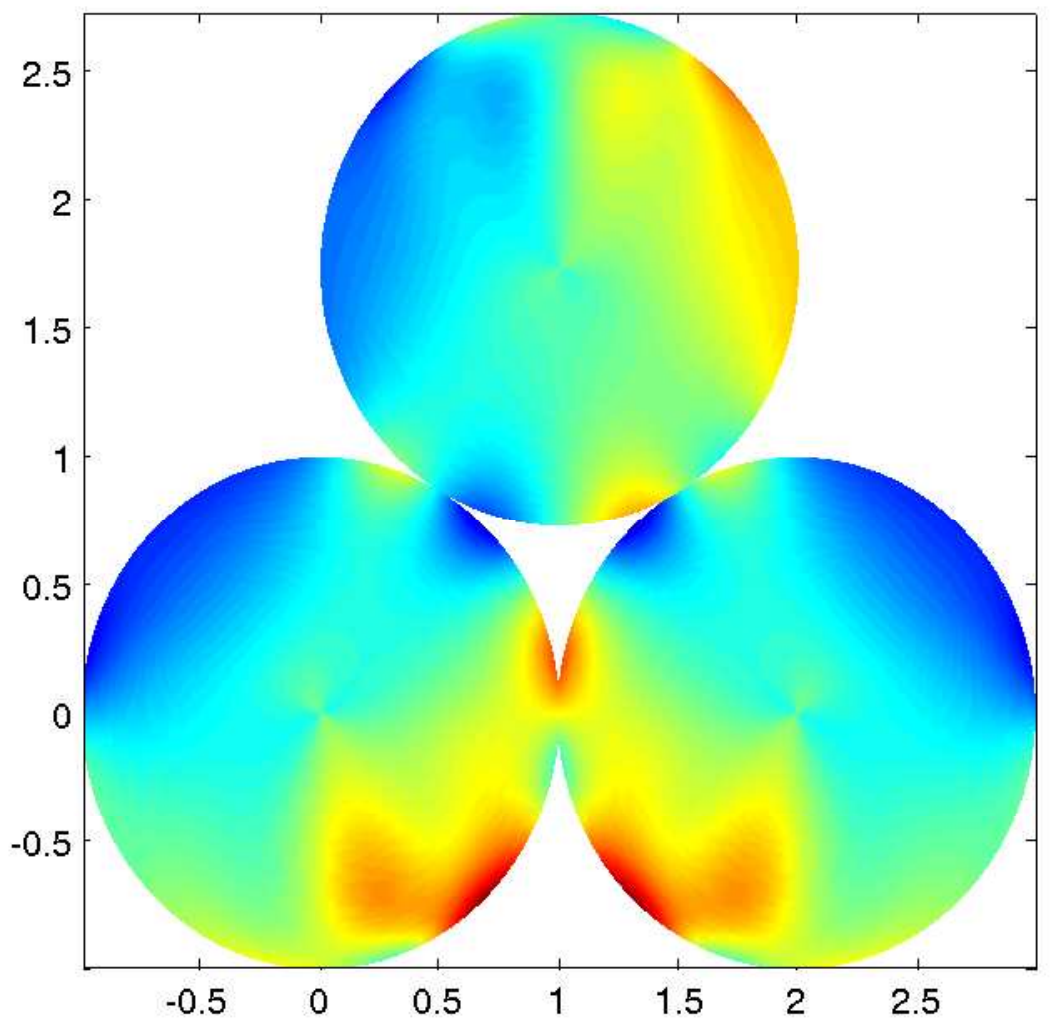

Figure 7: Shear stress distribution three disks. Traction is specified on the boundary of the system and displacement are given at the contact points 


\section{CONCLUSIONS}

The Lennard-Jones[5] model was our starting point to study the normal force between a pair of particles. The models of Ciamarra[1] and Cundall and Stack[2] are derived from equivalent physical principles, but, as we can see, the forces are modeled differently. This gives rise to differences in their numerical implementation. The Ciamarra model has a simpler form and an easier numerical implementation. We used the latter for our numerical implementation. Based on our numerical results, we have verified its computational efficiency.

Future research is recommended for generating the 'ghost' boundary particles. There is a possible relationship between elasticity of the boundary media to the density of the particles placed on the boundary. This relationship might be: the higher the density of the particles on the boundary, the more elastic the media. For example, if the media is porous or has holes in it, then it would make sense to make the particles less dense with spaces between them.

We considered a a simplified two-particle system with one stationary and one moving particle. When the moving particle is $d$ distance away from the stationary particle, a timestep

$$
\delta t<\frac{1}{\left|\sqrt{\left(\frac{48 \epsilon \sigma^{6}}{m}\right)\left(\frac{-13 \sigma^{6}}{d^{14}}+\frac{7}{2 d^{8}}\right)}\right|}
$$

will ensure that the leapfrog scheme is absolutely stable.

Future work will focus on extending this upper bound to more complex systems.

We solved the problem of finding stress distribution on a single disk and used this to solve the problem of a system with three disks. The approach we took in our investigation can be extended to systems of $n$ disks. Finally, we note that in contrast to previous works, we are taking a first step toward deriving a physically- based rather that an empirically- based force law to solve the problem of the flow of elastic granular materials.

\section{ACKNOWLEDGMENTS}

The work described herein was performed as part of the workshop "Mathematical Modeling in Industry IX" at the Institute for Mathematics and Applications (IMA) in Minneapolis, MN. We thank the organizers of the workshop, Prof. Fadil Santosa, Prof. Rich Braun and Prof. Fernando Reitich, for providing the opportunity to pursue this work. The IMA is thanked for financial support and for providing a wonderful working environment that contributed to the success of this project. PF performed this work under the auspices of the U.S. Department of Energy by University of California Lawrence Livermore National Laboratory under contract No. W-7405-Eng-48. 


\section{References}

[1] Massimo Pica Ciamarra, Antonio H. Lara, Andrew T. Lee, Daniel I. Goldman, Inna Vishik, and Harry L. Swinney. Dynamics of drag and force distributions for projectile impact in a granular medium. Physical Review Letters, 92(19):194301, 2004.

[2] P. A. Cundall and O. D. L. Strack. A discrete numerical model for granular assemblies. Geotechnique, 29:47-65, 1979.

[3] D. Howell, R. P. Behringer, and C. Veje. Stress fluctuations in a 2D granular couette experiment: A continuous transition. Phys. Rev. Lett., 82:5241-5244, 1999.

[4] B. Muth, M.-K. Muller, P. Eberhard, and S. Luding. Collision detection and administration for many colliding bodies. July 2002.

[5] D. C. Rapaport. The art of molecular dynamics simulation. Cambridge University Press, Cambridge, UK, 1st edition, 1995. 
Approved for public release; further dissemination unlimited 


$$
\mid
$$

